\title{
Spectrum of Thrombotic Disorders in Soldiers Serving in High-altitude Areas
}

\author{
Sandeep Kumar ${ }^{1}$, Niket Verma ${ }^{2}$, Sandeep Thareja ${ }^{3}$, Nalin K Mahesh ${ }^{4}$, Narendra Kotwal ${ }^{5}$, Sanjeev Kumar ${ }^{6}$, \\ Muthukrishnan Jayaraman ${ }^{7}$, Ashwin Mahesh ${ }^{8}$, Sourya S Mohakuda ${ }^{9}$
}

\begin{abstract}
Background: Indian soldiers serve in extreme climatic conditions at uninhabitable altitudes. Only few studies and case reports have described various thrombotic disorders in soldiers serving in high altitude (HA). It is postulated that there is activation of intravascular coagulation cascade and factors such as low temperatures, dehydration, polycythemia, stress, and hypoxia have been implicated.

Aim: To study the spectrum of thrombotic disorders occurring in soldiers serving in high-altitude areas (>10,000 feet).

Setting: The study was conducted at a tertiary-level referral centre for troops deployed in HA regions. The study involved a retrospective analysis of records of patients presenting with thrombotic disorders while being posted to HA. The duration of the study was between February 2012 and March 2014.

Materials and methods: Diagnosis, patient particulars, all investigations (including the thrombophilia workup), and treatment given were recorded. Patients with any pre-existing diseases were excluded from the study. Descriptive statistics were used for data analysis.

Results: Totally, 69 male patients were presented with thrombotic disorders during the study period. Only 30 (43.4\%) patients had pulmonary embolism, 18 (26\%) cerebral venous thrombosis, 9 (13\%) acute coronary syndrome, 7 (10.1\%) deep vein thrombosis, 4 (5.7\%) arterial stroke, and $1(1.4 \%)$ had retinal vein thrombosis. An estimated 14 (20.2\%) were smokers and 3 (4.3\%) patients were highlanders. The procoagulant workup was positive in 10 (14.4\%) patients, and 34 (49.2\%) patients had polycythemia. The mean duration of stay at HA was $5 \pm 2$ months. There was no mortality.

Conclusion: Only $14.4 \%$ of patients showed prothrombotic states. The role of causative factors and the unknown prothrombotic condition in HA needs further investigation by trials involving a larger study population.

Keywords: Altitude, Soldiers, Thrombosis.

Journal of Medical Academics (2019): 10.5005/jp-journals-10070-0027
\end{abstract}

\section{INTRODUCTION}

Deployment in extremes of high altitude (HA) in the Himalayas is an exclusive challenge to which Indian troops are exposed. ${ }^{1}$ The extremes of climatic condition at HA such as low oxygen and low temperatures are detrimental to human survival but detailed implications have not been validated till date by clinical trials. Troops are more vulnerable to adverse health outcomes in such extreme conditions because, by virtue of being deployed in a conflict zone, they are also exposed to physical and psychological stress, dehydration, dietary restrictions, and prolonged immobilization. HA-related illnesses (including thrombotic disorders at HA) are not well studied despite finding mention in various case reports and isolated observational small studies. A variety of thrombotic disorders have been reported in troops serving in high altitude, ranging from deep vein thrombosis, portal vein thrombosis, and to pulmonary embolism. ${ }^{1-3}$ It postulated that in the absence of hereditary prothrombotic states, conditions such as hypoxia, extremely cold temperatures, prolonged immobilization, and dehydration are major risk factors for increased vascular thrombosis in HA. Moreover, in soldiers deployed in such areas, the role of psychological stress and dietary deficiencies is also implicated by some authors. As a result of these known and some unknown risk factors, there is activation of intravascular coagulation cascade and hence the various thrombotic presentations. ${ }^{2,4,5}$ We studied 69 consecutive patients presenting with thrombotic disorders at a hospital, which serves as a tertiary-level referral centre for the soldiers being evacuated from high-altitude regions of northern India.
$1-4,7,8$ Department of General Medicine, Army College of Medical Sciences, New Delhi, India

${ }^{5}$ Department of General Medicine, Army Hospital Research and Referral, New Delhi, India

${ }^{6}$ Armed Forces Clinic, New Delhi, India

${ }^{9}$ Department of General Medicine, Armed Forces Medical College, Pune, Maharashtra, India

Corresponding Author: Niket Verma, Department of General Medicine, Army College of Medical Sciences, New Delhi, India, Phone: +919818340326, e-mail: drniketverma@gmail.com

How to cite this article: Kumar S, Verma N, et al. Spectrum of Thrombotic Disorders in Soldiers Serving in High-altitude Areas. J Med Acad 2019;2(1):17-19.

Source of support: Nil

Conflict of interest: None

\section{Materials and Methods}

The study was conducted at a tertiary-level referral centre for the troops deployed in high-altitude (HA) regions in northern India. The study involved retrospective analysis of records of consecutive patients presenting with various thrombotic disorders while being posted to HA (>10,000 feet) and presenting between February 2012 and March 2014. Approval of Institutional Ethical Committee was taken. No consent was needed from the patients as the study only involved analysis of records. Patients with any pre-existing diseases were excluded from the study. Detailed clinical data 
of patients (including place of residence, smoking history, and pre-morbidities) were recorded. Diagnosis, patient particulars, all investigations (including the thrombophilia workup), and treatment given were recorded. Patients with any pre-existing conditions which predispose to increased thrombosis were excluded from the study. Descriptive statistics were used for analysis of the data.

Routine hematological and biochemical parameters (including lipid profile and blood sugar) were recorded. Pulmonary embolism (PE) was diagnosed by pulmonary CT angiography, cortical venous thrombosis (CVT) by MR venography, stroke by MRI brain, acute coronary syndrome by ECG and troponin evaluation, deep vein thrombosis (DVT) by limb Doppler, and retinal vein thrombosis by fluorescein angiography.

Investigations to detect prothrombotic states were done at presentation and after 6 months of therapy. Prothrombotic state workup included antinuclear antibodies (ANA), rheumatoid factor (RF), anticardiolipin antibody (ACLA), protein $C$ and $S$ and antithrombin III deficiency, as well as antibodies to hepatitis $B$ and $C$ and syphilis. All patients were provided therapy by concerned specialists as per standard guidelines.

\section{Results}

A total of 69 male patients were presented with various thrombotic disorders during the study period. There were no females in the patients.

Mean age of the patients was $31 \pm 4.2$ years. Baseline patient characteristics are tabulated in Table 1. An estimated 14 (20.2\%) were smokers and $3(4.3 \%)$ patients were highlanders. The mean duration of stay at HA was $5 \pm 2$ months. The mean altitude of deployment was $11,830 \pm 320$ feet, ranging from 10,000 feet to 21,000 feet.

Of the 69 patients, 30 (43.4\%) patients had pulmonary embolism, 18 (26\%) had cerebral venous thrombosis, 9 (13\%) had acute coronary syndrome, 7 (10.1\%) had deep vein thrombosis, $4(5.7 \%)$ had arterial stroke, and $1(1.4 \%)$ had retinal vein thrombosis. Table 2 depicts various thrombotic diseases seen in our patients.

Procoagulants workup was positive in $10(14.4 \%)$ patients and 34 (49.2\%) patients had polycythemia ( $\mathrm{Hb}>17 \mathrm{~g} / \mathrm{dL}$ ). Table 3 describes various prothrombotic states detected in our patient.

Table 1: Baseline patient characteristics

\begin{tabular}{ll}
\hline Parameter & Number $n=69(100 \%)$ \\
\hline Males & $69(100 \%)$ \\
Mean age & $31 \pm 4.2$ years \\
Duration of stay at HA & $5 \pm 2$ months \\
Mean altitude & $11,830 \pm 320$ feet \\
Highlanders & $3(4.3 \%)$ \\
Smokers & $14(20.2 \%)$ \\
\hline
\end{tabular}

Table 2: Various thrombotic diseases in study population

\begin{tabular}{ll}
\hline Thrombotic diseases & Number $n=69(100 \%)$ \\
\hline Pulmonary embolism & $30(43.4 \%)$ \\
Cerebral venous thrombosis & $18(26 \%)$ \\
Acute coronary syndrome & $9(13 \%)$ \\
Deep vein thrombosis & $7(10.1 \%)$ \\
Stroke & $4(5.7 \%)$ \\
Retinal vein thrombosis & $1(1.4 \%)$ \\
\hline
\end{tabular}

Table 3: Prothrombotic workup profile of patients

\begin{tabular}{ll}
\hline Parameter & Number 10 (100\%) \\
\hline Hyper homocystinemia & $3(30 \%)$ \\
Protein C deficiency & $1(10 \%)$ \\
Protein S deficiency & $2(20 \%)$ \\
ANA & $1(10 \%)$ \\
ACLA & 0 \\
Antithrombin III deficiency & $3(30 \%)$ \\
\hline
\end{tabular}

Of the total patients, 3 (30\%) had hyperhomocystinemia, 3 (30\%) had antithrombin III deficiency, $2(20 \%)$ had protein S deficiency, $1(10 \%)$ had protein C deficiency, and 1 (10\%) patient was ANA positive. None of the patients tested positive for anti-cardiolipin antibody.

\section{Discussion}

Our study is a retrospective analysis of records of soldiers who are presented with thrombotic diseases while being posted in HA regions. Despite limitations of the study design, our study has brought out the fact that arterial as well as venous thrombosis occur at an increased rate in soldiers deployed in HA regions. Moreover, these soldiers are young and have high physical fitness standards and are generally free from any chronic ailments or functional limitations. A detailed basic physical fitness medical examination is held for such troops before recruitment into military service as well as before induction into high-altitude regions. To the best of our knowledge, there is no other study in the India that describes the constellation of thrombotic disorders in soldiers while posted in high-altitude areas. Our study has revealed that hereditary prothrombotic states do not play a major role in causation of high-altitude-related thrombosis as only $14.4 \%$ patient had positive prothrombotic state screening. Similar results were also seen in high-altitude-induced pulmonary embolism by Dutta et al. ${ }^{2}$ A previous study has shown that hereditary prothrombotic states were seen in about $30 \%$ of patients who had pulmonary embolism without exposure to high altitude. ${ }^{6} \mathrm{~A}$ low prevalence of prothrombotic states in our patients is explained possibly by the fact that our study had young healthy serving soldiers who were free from any chronic ailments.

These serving young soldiers are highly selected men who are considered free from any mental or physical ailments. However, being deployed in warzones with harsh climatic conditions and away from family members do predispose to mental stress. Mental stress has been implicated in occurrence of thrombosis at higher rates associated with higher D-dimers level and increased sympathetic activity. ${ }^{7}$ Though the militaries of all nations provide scientifically designed meals to their troops to cater for body requirement in such harsh conditions, there is still a lack of fresh fruits and vegetables and dehydration has been suggested to contribute toward accelerated thrombosis. ${ }^{8}$ Hypoxia is another important factor believed to be responsible for increased thrombotic manifestation at HA. Hypoxia causes activation of intravascular coagulation cascade and also leads to polycythemia by stimulating erythropoietin. ${ }^{9,10}$

Limitations of our study is small sample size and only male population. A well-designed trial with a larger sample size is suggested by authors to investigate into high-altitude-related thrombotic diseases. 


\section{Conclusion}

Both arterial and venous thromboses definitely occur at a higher rate in HA areas. Hereditary prothrombotic states are seen to be responsible for such thrombosis only in minimal cases. The role of low temperatures, diet, stress, hypoxia, etc., in thrombosis needs investigation. The unknown prothrombotic condition in HA need to be further studied by well-planned trials involving a larger study population.

\section{REFERENCES}

1. Acosta MP. High altitude warfare: The Kargil conflict and the future. Masters of Arts Thesis, Naval Post Graduate School, Monterey, California, June 2003.

2. Dutta $V$, Singh $R$, et al. Profile of pulmonary embolism in service personnel posted at high altitude area. Indian Heart J 2018 May-Jun; 70(3):427-429. DOI: 10.1016/j.ihj.2017.08.002.

3. Anand AC, Saha A, et al. Symptomatic portal system thrombosis in soldiers due to extended stay at extreme high altitude.J Gastroenterol Hepatol 2005;20:777-783. DOI: 10.1111/j.1440-1746.2005.03723.x.
4. Khalil KF, Saeed W. Pulmonary embolism in soldiers serving at high altitude. J Coll Physicians Surg Pak 2010 Jul;20(7):468-471. DOI: 07.2010/JCPSP.468471.

5. Martinelli I, Bucciarelli $P$, et al. Thrombotic risk factors: basic pathophysiology. Crit Care Med 2010;38:S3-S9. DOI: 10.1097/ CCM.0b013e3181c9cbd9.

6. West JB. The physiological basis of high-altitude diseases. Ann Intern Med 2004;141:789-800. DOI: 10.7326/0003-4819-141-10-20041116000010.

7. Turan $\mathrm{O}$, Ündar B, et al. Investigation of inherited thrombophilias in patients with pulmonary embolism. Blood Coagul Fibrinolysis 2013;24(2):140-149. DOI: 10.1097/MBC.0b013e328359db0e.

8. Wirtz HP, Bärtschi C, et al. Effect of oral melatonin on the procoagulant response to acute psychosocial stress in healthy men: a randomized placebo controlled study. J Pineal Res 2008;44(4):358-365. DOI: 10.1111/j.1600-079X.2007.00535.x.

9. Gupta R, Joshi P, et al. Epidemiology and causation of coronary heart disease and stroke in India. Heart 2008;94:16-26. DOI: 10.1136/ hrt.2007.132951.

10. Bärtsch P. How thrombogenic is hypoxia? JAMA 2006;295:2297-2299. DOI: 10.1001/jama.295.19.2297. 